\title{
Macrófitas de la cuenca del Río Elqui, zonA SEMIÁRIDA DE CHILE
}

\author{
Macrophytes of the Elqui River basin, semiarid zone of ChILE
}

\author{
Jonathan Urrutia-Estrada ${ }^{1,2 *}$ y Mario Romero-Mieres ${ }^{3}$ (D)
}

1. Laboratorio de Invasiones Biológicas, Facultad de Ciencias Forestales, Universidad de Concepción, Concepción, Chile.

2. Instituto de Ecología y Biodiversidad (IEB), Concepción, Chile.

3. Laboratorio de Ecología Aplicada y Biodiversidad, Departamento de Ciencias Ambientales, Facultad de Recursos Naturales, Universidad Católica de Temuco, Temuco, Chile.

*jurrutiaestrada@gmail.com

Citar este artículo

URRUTIA-ESTRADA, J. \& $M$. ROMERO-MIERES. 2022. Macrófitas de la cuenca del río Elqui, zona semiárida de Chile Bol. Soc. Argent. Bot. 57: 101-116.

DOI: https://doi. org/10.31055/1851.2372.v57. n1.32741

Recibido: 14 Abr 2021

Aceptado: 16 Nov 2021

Publicado en línea: 20 Feb 2022

Publicado impreso: 31 Mar 2022

Editora: Carolina I. Calviño (DD

ISSN versión impresa 0373-580X

ISSN versión on-line 1851-2372

\section{SUMMARY}

Background and aims: The Elqui river basin is located north of the Coquimbo Region, in the semiarid zone of Chile. This body of water is particularly important due to the economic activities it supports and its high environmental vulnerability. The objective of this study was to determine the diversity and floristic composition of macrophytes of the Elqui river basin.

M\&M: Twenty sampling stations with plots of $1 \mathrm{~m}^{2}$ were distributed in the three sub-basins that make up the study area. Inside each plot, all plant species were recorded along with their respective abundances. In addition, in each station some environmental variables were measured, which were analyzed together with the biological data.

Results: A total of 51 macrophyte species and one alga were identified, with dominance of native elements with emergent habit. Statistical analyses indicate moderate differences regarding the floristic composition of the three sub-basins compared; these are mainly concentrated in the Turbio river. The analysis of the environmental data indicates that the variables that most influence the composition of species in the study area are: altitude, total suspended solids, redox potential and water depth.

Discussion: Studies on macrophytes at the hydrographic basin level are practically null in Chile. This type of information increases knowledge on biodiversity and allows a more efficient management of continental aquatic systems.

\section{KEY WORDS}

Altitude, aquatic plants, floristic diversity, river systems.

\section{REsumen}

Introducción y objetivos: La cuenca del río Elqui está situada al norte de la Región de Coquimbo, en la zona semiárida de Chile. Este cuerpo de agua es particularmente importante por las actividades económicas que sustenta y por su alta vulnerabilidad ambiental. El objetivo del presente estudio fue determinar la diversidad y composición florística de macrófitas de la cuenca del río Elqui.

M\&M: Se establecieron 20 estaciones de muestreo con parcelas de $1 \mathrm{~m}^{2}$ distribuidas en las tres subcuencas que conforman el área de estudio. Al interior de cada una de ellas, se registraron todas las especies de plantas presentes junto a sus respectivas abundancias. Además, en cada estación se midieron algunas variables ambientales, las que fueron analizadas junto con los datos biológicos.

Resultados: Se identificaron 51 especies de macrófitas y un alga, con dominancia de elementos nativos y de hábito emergente. Los análisis estadísticos señalan leves diferencias en cuanto a la composición florística que presentan las tres subcuencas comparadas, las cuales se concentran principalmente en el río Turbio. El análisis de los datos ambientales señala que las variables que más influyen en la composición de especies del área de estudio son: altitud, sólidos suspendidos totales, potencial redox y profundidad del agua.

Discusión: Los estudios relativos a macrófitas a nivel de cuenca hidrográfica son prácticamente nulos en Chile. Este tipo de información incrementa el conocimiento de la biodiversidad y permite realizar un manejo más eficiente de los sistemas acuáticos continentales.

Palabras clave

Altitud, diversidad florística, plantas acuáticas, sistemas fluviales. 


\section{INTRODUCCIÓN}

En Chile, los sistemas lóticos (aguas corrientes) han recibido menor atención en cuanto a investigación que sus contrapartes lacustres, marinas o terrestres (Palma et al., 2013). Esta diferencia se exacerba al considerar los sistemas fluviales de regiones áridas y semiáridas, ya que se encuentran dentro de los ecosistemas menos estudiados del planeta (Davies et al., 1994). En estos ambientes, el balance de agua suele ser negativo, a raíz de las circunstancias climáticas menos favorables (Vidal-Abarca et al., 2004). La heterogeneidad espacio-temporal a distintas escalas que presentan estos ríos es de tal magnitud, que supone un reto científico para entender las claves de su estructura y funcionamiento (Gómez et al., 2001).

La información biótica disponible para las cuencas hidrográficas de la zona semiárida de Chile, está mayormente almacenada en estudios de impacto ambiental, llevados a cabo principalmente por consultoras (Palma et al., 2013). En particular, los estudios florísticos realizados en cuerpos de agua continentales se presentan mayormente en la zona centro-sur del país (Hauenstein et al., 2011; San Martín et al., 2011; Urrutia et al., 2012; Sandoval et al., 2016; Hauenstein \& Urrutia-Estrada, 2018; Baeza et al., 2019, entre otros) y parcialmente en la región mediterránea (Ramírez \& San Martín, 1984; Ramírez et al., 1987; San Martín et al., 2001). Consecuentemente, existe un vacío de información en un área tan importante y vulnerable como la zona semiárida de Chile. En este contexto, el río Elqui sostiene importantes actividades económicas asociadas a su cuenca, donde se destacan la industria agrícola y minera (Guevara et al., 2006). Del mismo modo, presenta importantes condiciones de vulnerabilidad debido a su posición transversal meridional respecto al Desierto de Atacama, con un aporte pluviométrico mayoritariamente andino nival $\mathrm{y}$ un gradiente altitudinal desde los 0 a los 5000 msnm (Cepeda et al., 2008). Las implicancias de lo descrito anteriormente para la cuenca del río Elqui se traducen en una posición rodeada de un interfluvio árido, acompañada de una elevada irregularidad del régimen de precipitaciones, propio de zonas semiáridas. En este sentido, el comienzo de las lluvias luego de una prolongada estación seca hace que el suelo se encuentre reseco y desprovisto de una cubierta herbácea, por lo cual resulta fácilmente erosionable y disgregable. Más aún, a esta latitud se presenta el punto más angosto de Chile, por lo que, la corta distancia entre la cordillera y el mar provoca el desarrollo de un territorio abrupto, con fuertes pendientes y un relieve mayormente irregular (Conte, 1986). Las aguas del río Elqui son el principal sustento hídrico de la actividad humana que se desarrolla preferentemente en la zona costera, en las ciudades de La Serena y Coquimbo. Más del $90 \%$ de la precipitación anual se concentra en los meses de invierno y ocurre en forma de nieve en el ámbito cordillerano. Las acumulaciones nivales actúan como un valioso embalse natural que libera su caudal en primavera, permitiendo regar el valle y sus afluentes y abastecer las necesidades de la población, posibilitando el desarrollo de la economía local.

Las macrófitas son esenciales en la estructura y funcionamiento de muchos ecosistemas. Como productores primarios tienen un importante rol en el ciclo de nutrientes, además de representar un vínculo entre el agua y el sedimento (Wetzel, 2001). Mejoran la calidad del agua, reducen la erosión y turbidez (Madsen et al., 2001), e influyen de manera considerable en la dinámica del oxígeno disuelto en zonas potamónicas (Desmet et al., 2011). El escenario de escasez hídrica que enfrenta Chile pone un énfasis especial en conocer la biodiversidad que albergan los cuerpos de agua, especialmente en la zona semiárida del país. En consideración a lo anterior, el objetivo del presente estudio es determinar y analizar la diversidad y composición de la flora de macrófitas de la cuenca del río Elqui.

\section{Materiales y Métodos}

\section{Área de estudio}

La cuenca hidrográfica del río Elqui se sitúa en el sector norte de la Región de Coquimbo, entre los paralelos $29^{\circ} 35^{\prime}$ y $30^{\circ} 20^{\prime}$ de latitud sur, y los meridianos $71^{\circ} 18^{\prime}$ y $69^{\circ} 55^{\prime}$ de longitud oeste, abarcando una superficie aproximada de 9826 $\mathrm{km}^{2}$ (Fig. 1). A $815 \mathrm{msnm}$, dos kilómetros aguas arriba de la localidad de Rivadavia, se unen los ríos Turbio y Claro, provenientes desde el oriente y el sur respectivamente, dando origen al río Elqui. Desde Rivadavia, el río principal se desarrolla casi en dirección este-oeste y prácticamente no recibe 


\section{J. Urrutia-Estrada y M. Romero-Mieres - Macrófitas de la cuenca del río Elqui}
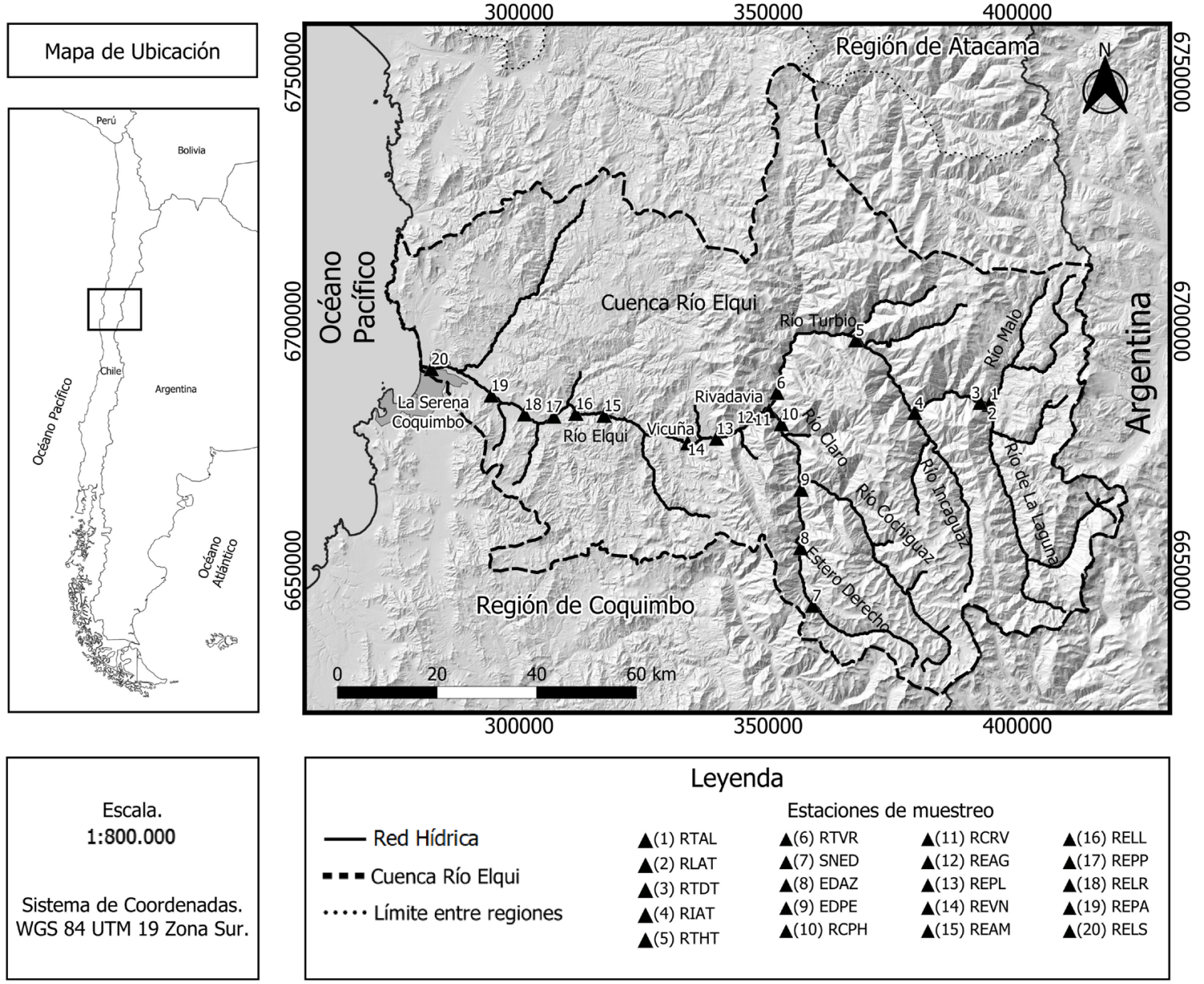

Fig. 1. Ubicación de la cuenca del río Elqui en la Región de Coquimbo, Chile, indicando las estaciones de muestreo en las tres subcuencas estudiadas. Subcuenca del río Turbio (estaciones 1-6), subcuenca del río Claro (estaciones 7-11), subcuenca del río Elqui (estaciones 12-20). Las abreviaciones de las estaciones de muestreo siguen la Tabla 1.

afluentes, salvo varias quebradas de considerable desarrollo, pero normalmente secas y que sólo le aportan agua en caso de lluvia directa en los años muy húmedos. La precipitación media anual en la cuenca del río Elqui se ubica en el rango de 60 a $170 \mathrm{~mm}$, con una tendencia de mayor precipitación a mayor altitud. Para efectos de ordenación y análisis de datos, el área de estudio se dividió en tres subcuencas: río Claro, río Turbio y río Elqui.

\section{Recopilación y tabulación de datos}

La recopilación de datos se realizó en el mes de noviembre de 2019, en 20 estaciones de muestreo distribuidas en toda el área de estudio (Fig. 1); cinco en la subcuenca del río Claro, seis en la subcuenca del río Turbio y nueve en la subcuenca del río Elqui (Tabla 1). La diferencia en el número de estaciones de muestreo radica en la distinta morfología de hábitat que presentó cada subcuenca. En cada estación se establecieron dos transectos de $40 \mathrm{~m}$ de largo de manera paralela a la orilla del río, uno desde la línea de costa hacia el exterior y otro hacia el interior, lo que permitió abarcar las zonas que se inundan de manera permanente y temporal. Sobre cada transecto se levantaron al menos 20 parcelas de 1 $\mathrm{m}^{2}$, dispuestas de manera equidistante (Steubing 
Tabla 1. Código, descripción, subcuenca y ubicación en coordenadas UTM (zona 19J) de las estaciones de muestreo de la cuenca del río Elqui.

\begin{tabular}{|c|c|c|c|c|}
\hline Código & Descripción & Subcuenca & UTM E & UTM N \\
\hline RTAL (1) & Río Toro antes de confluencia con río La Laguna & Turbio & 394578 & 6683551 \\
\hline RLAT (2) & Río La Laguna antes de confluencia con río Toro & Turbio & 394367 & 6683358 \\
\hline RTDT (3) & Río Turbio después de confluencia entre río Toro y río La Laguna & Turbio & 392542 & 6683770 \\
\hline RIAT (4) & Río Incaguaz antes de confluencia con río Turbio & Turbio & 379397 & 6681733 \\
\hline RTHT (5) & Río Turbio en Huanta & Turbio & 367561 & 6696344 \\
\hline RTVR (6) & Río Turbio en Varillar & Turbio & 351751 & 6685774 \\
\hline SNED (7) & Santuario de la Naturaleza Estero Derecho & Claro & 358925 & 6643083 \\
\hline EDAZ (8) & Estero Derecho en Alcohuaz & Claro & 356508 & 6654644 \\
\hline EDPE (9) & Estero Derecho en Pisco Elqui & Claro & 356590 & 6666376 \\
\hline $\mathrm{RCPH}(10)$ & Río Claro en Paihuano & Claro & 352644 & 6679506 \\
\hline RCRV (11) & Río Claro en Rivadavia & Claro & 350164 & 6682099 \\
\hline REAG (12) & Río Elqui en Algarrobal & Elqui & 347089 & 6680729 \\
\hline REPL (13) & Río Elqui en Peralillo & Elqui & 339624 & 6676606 \\
\hline REVN (14) & Río Elqui en Vicuña & Elqui & 333833 & 6675661 \\
\hline REAM (15) & Río Elqui en Almendral & Elqui & 317066 & 6681207 \\
\hline RELL (16) & Río Elqui en puente El Molle & Elqui & 311398 & 6681587 \\
\hline REPP (17) & Río Elqui en puente Pelicana & Elqui & 306954 & 6681015 \\
\hline RELR (18) & Río Elqui en Las Rojas & Elqui & 301110 & 6681466 \\
\hline REPA (19) & Río Elqui en puente Altovalsol & Elqui & 294432 & 6685262 \\
\hline RELS (20) & Río Elqui en La Serena & Elqui & 282379 & 6690547 \\
\hline
\end{tabular}

et al., 2002). En cada parcela se registró la riqueza (número de especies de macrófitas), y se estimó la abundancia (cobertura) de cada una de ellas expresada en porcentaje del área cubierta (Mueller-Dombois \& Ellenberg, 1974). Las especies que no pudieron ser identificadas en terreno, fueron coleccionadas, etiquetadas y prensadas. Posteriormente se identificaron en gabinete a través de literatura especializada (e.g. Ramírez et al., 1982; Rodríguez \& Dellarossa, 1998; DiTomaso \& Healy, 2003 y Urrutia et al., 2017a). Las muestras coleccionadas fueron depositadas en el Herbario de la Universidad Católica de Temuco.

Cabe señalar que para efectos del presente estudio se utilizó el concepto de macrófita en un sentido amplio (Ramírez \& San Martín, 2018), es decir, a las plantas vasculares acuáticas (aquellas que viven en el agua, parcial o totalmente sumergidas). Esto permitió incluir algunas especies ruderales comunes en la ribera de los cuerpos de agua. Además, de manera excepcional se incorporó una especie de alga carófita (Chara vulgaris L.), debido a la abundancia que presentó en algunas estaciones de muestreo. La clasificación, nomenclatura y el origen geográfico de las especies se determinó según Urrutia et al. (2017a), Rodríguez et al. (2018), y Zuloaga et al. (2019). El trabajo en terreno permitió confeccionar una tabla de presencia/cobertura de las especies presentes en cada estación de muestreo, y un catálogo florístico, en el cual se incluyen todas las especies de macrófitas identificadas. Para cada una de ellas se indica su nombre científico, autores de los taxones, familia botánica, nombre común, origen geográfico y hábito de crecimiento (Tabla 2). Esto último fue tomado de Sculthorpe (1967), cuya clasificación se divide en emergentes, natantes, flotantes libres y sumergidas. Con el fin de determinar las especies más relevantes del área 


\section{J. Urrutia-Estrada y M. Romero-Mieres - Macrófitas de la cuenca del río Elqui}

Tabla 2. Listado de macrófitas registradas en la cuenca del río Elqui, indicando nombre científico de

la especie y familia a la que pertenece, nombre común, status en cuanto al origen de las especies

en el Cono Sur de Sudamérica (EN: endémico, EX: exótico, NA: nativo) y hábito de crecimiento (E: emergente, F: flotante libre, N: natante, S: sumergido).

\begin{tabular}{|c|c|c|c|c|}
\hline Nombre científico & Familia & Nombre común & Status & Hábito \\
\hline Alisma lanceolatum With. & Alismataceae & Llantén de agua & EX & $E$ \\
\hline Apium nodiflorum (L.) Lag. & Apiaceae & Apio de agua & EX & $\mathrm{E}$ \\
\hline Hydrocotyle modesta Cham. \& Schltdl. & Apiaceae & Sombrerito de agua & NA & E \\
\hline Hydrocotyle ranunculoides L.f. & Apiaceae & Hierba de la plata & EX & $\mathrm{N}$ \\
\hline Hydrocotyle verticillata Thunb. & Apiaceae & Sombrerito de agua & NA & $E$ \\
\hline Baccharis sagittalis (Less.) DC. & Asteraceae & Verbena de tres esquinas & NA & E \\
\hline Cotula coronopifolia L. & Asteraceae & Botón de oro & EX & $\mathrm{E}$ \\
\hline Azolla filiculoides Lam. & Azollaceae & Flor del pato & EX & $\mathrm{F}$ \\
\hline Cardamine volckmannii Phil. & Brassicaceae & Berrillo & NA & E \\
\hline Nasturtium officinale R. Br. & Brassicaceae & Berro europeo & EX & E \\
\hline Rorippa sylvestris (L.) Besser & Brassicaceae & Pata de laucha & EX & E \\
\hline Cerastium humifusum Cambess. & Caryophyllaceae & - & NA & E \\
\hline Chara vulgaris $\mathrm{L}$. & Characeae & Alga candelabro & EX & S \\
\hline Carex acutata Boott & Cyperaceae & Cortadera & EN & $E$ \\
\hline Carex gayana E. Desv. & Cyperaceae & Pasto de vega & NA & E \\
\hline Cyperus eragrostis Lam. & Cyperaceae & Cortadera & NA & $\mathrm{E}$ \\
\hline Eleocharis pachycarpa E. Desv. & Cyperaceae & Rume & NA & E \\
\hline $\begin{array}{l}\text { Eleocharis pseudoalbibracteata S. González \& } \\
\text { Guagl. }\end{array}$ & Cyperaceae & Rume & NA & $\mathrm{E}$ \\
\hline $\begin{array}{l}\text { Phylloscirpus acaulis (Phil.) Goetgh. \& D.A. } \\
\text { Simpson }\end{array}$ & Cyperaceae & - & NA & $\mathrm{E}$ \\
\hline $\begin{array}{l}\text { Rhodoscirpus asper (J. Presl \& C. Presl) Léveillé- } \\
\text { Bourret, Donadío \& J.R. Starr }\end{array}$ & Cyperaceae & Totora & NA & $\mathrm{E}$ \\
\hline $\begin{array}{l}\text { Schoenoplectus americanus (Pers.) Volkart ex } \\
\text { Schinz \& R. Keller }\end{array}$ & Cyperaceae & Totora & NA & $\mathrm{E}$ \\
\hline Schoenoplectus californicus (C.A. Mey.) Soják & Cyperaceae & Totora & NA & E \\
\hline Equisetum bogotense Kunth & Equisetaceae & Hierba de la plata & NA & E \\
\hline Equisetum giganteum $\mathrm{L}$. & Equisetaceae & Cola de caballo & NA & E \\
\hline Myriophyllum aquaticum Verdc. & Haloragaceae & Pinito de agua & EX & S \\
\hline Myriophyllum quitense Kunth & Haloragaceae & Loroma & NA & s \\
\hline $\begin{array}{l}\text { Limnobium laevigatum (Humb. \& Bonpl. ex Willd.) } \\
\text { Heine }\end{array}$ & Hydrocharitaceae & Hierba guatona & EX & $\mathrm{F}$ \\
\hline $\begin{array}{l}\text { Juncus balticus Willd. subsp. mexicanus (Willd. ex } \\
\text { Roem. \& Schult.) Snogerup }\end{array}$ & Juncaceae & Junquillo & NA & $\mathrm{E}$ \\
\hline Juncus pallescens Lam. & Juncaceae & Hierba de la vaca & NA & $\mathrm{E}$ \\
\hline Juncus procerus E. Mey. & Juncaceae & Junquillo & NA & E \\
\hline Mentha aquatica L. & Lamiaceae & Menta negra & EX & E \\
\hline Mentha pulegium $\mathrm{L}$. & Lamiaceae & Poleo & EX & E \\
\hline Ludwigia peploides (Kunth) P.H. Raven & Onagraceae & Clavito de agua & EX & $\mathrm{N}$ \\
\hline Habenaria pumila Poepp. & Orchidaceae & - & NA & E \\
\hline
\end{tabular}




\begin{tabular}{|lllll|}
\hline \multicolumn{1}{|c}{ Nombre científico } & \multicolumn{1}{c}{ Familia } & \multicolumn{1}{c}{ Nombre común } & Status & Hábito \\
Erythranthe depressa (Phil.) G.L. Nesom & Phrymaceae & Placa & EN & E \\
Erythranthe lutea (L.) G.L. Nesom. & Phrymaceae & Placa & NA & E \\
Veronica anagallis-aquatica L. & Plantaginaceae & No me olvides & EX & E \\
Agrostis capillaris L. & Poaceae & Chépica & EX & E \\
Arundo donax L. & Poaceae & Carrizo gigante & EX & E \\
Distichlis spicata (L.) Greene & Poaceae & Pasto salado & NA & E \\
Polypogon australis Brongn. & Poaceae & Cola de ratón & NA & E \\
Polygonum hydropiperoides Michx. & Polygonaceae & Duraznillo de agua & EX & E \\
Rumex conglomeratus Murray & Polygonaceae & Romaza & EX & E \\
Potamogeton pusillus L. & Potamogetonaceae & Huiro & EX & S \\
Stuckenia filiformis (Pers.) Boehm. & Potamogetonaceae & Canehuin & S \\
Zannichellia palustris L. & Potamogetonaceae & Cachudita del agua & EX & S \\
Samolus repens (J.R. Forst. \& G. Forst.) Pers. & Primulaceae & - & NA & E \\
Ranunculus peduncularis Sm. var. peduncularis & Ranunculaceae & Botón de oro & NA & E \\
Ranunculus repens L. & Ranunculaceae & Botón de oro & EX & E \\
Salix babylonica L. & Salicaceae & Sauce llorón & EX & E \\
Typha angustifolia L. & Typhaceae & Vatro & EX & E \\
Phyla nodiflora (L.) Greene & Verbenaceae & Tiqui-tiqui & NA & E \\
\hline
\end{tabular}

de estudio, se calculó el Valor de Importancia. Para esto, se registró la frecuencia de cada especie (número de estaciones presente), la que se indicó en números absolutos (FA) y en términos de frecuencia relativa (FR), indicando el porcentaje de frecuencia de cada especie tomando como 100 \% la sumatoria de FA. Posteriormente se anotaron los valores correspondientes a la cobertura absoluta de cada especie (CA), y su cobertura relativa (CR) o porcentaje de la cobertura total de cada especie, utilizando como $100 \%$ la sumatoria de las coberturas. Finalmente, al sumar la frecuencia y cobertura relativas de acuerdo a Wikum \& Shanholtzer (1978), se obtuvo el Valor de Importancia de cada especie.

\section{Variables ambientales}

Todas las variables ambientales fueron registradas in situ, previo a la recopilación de datos biológicos. En cada estación de muestreo se midieron parámetros de la columna de agua, del sedimento, del sustrato y del tipo topográfico. En la Tabla 3, se muestra el detalle de las 15 variables ambientales consideradas, la unidad de medida y el instrumental utilizado.

\section{Análisis estadístico de datos}

Para determinar la ordenación de las estaciones de muestreo en base a su composición florística, se llevó a cabo un análisis de escalamiento multidimensional no-métrico (nMDS) a partir de una matriz de similitud de Bray-Curtis. Además, se realizó un análisis de similitud (ANOSIM) de una vía y un test a posteriori de comparaciones pareadas, con el fin de determinar posibles diferencias estadísticas con base en la composición florística. Para ambas pruebas se definió como factor predictor la ubicación de la subcuenca (i.e. Turbio, Claro y Elqui).

Para determinar las variables ambientales que más influyen en la composición florística, se realizó un análisis BioEnv (Biota-Environment). En primer lugar, se realizó una prueba de Draftsman Plot, la cual permitió eliminar variables correlacionadas. Posteriormente, se trabajó con la matriz biológica (Tabla 4), y se confeccionó una matriz de datos ambientales (Tabla 3), en la cual se incluyeron 15 variables medidas en el trabajo de campo. Todos los análisis estadísticos se realizaron con el software Primer v.6 (Clarke \& Gorley, 2006). 


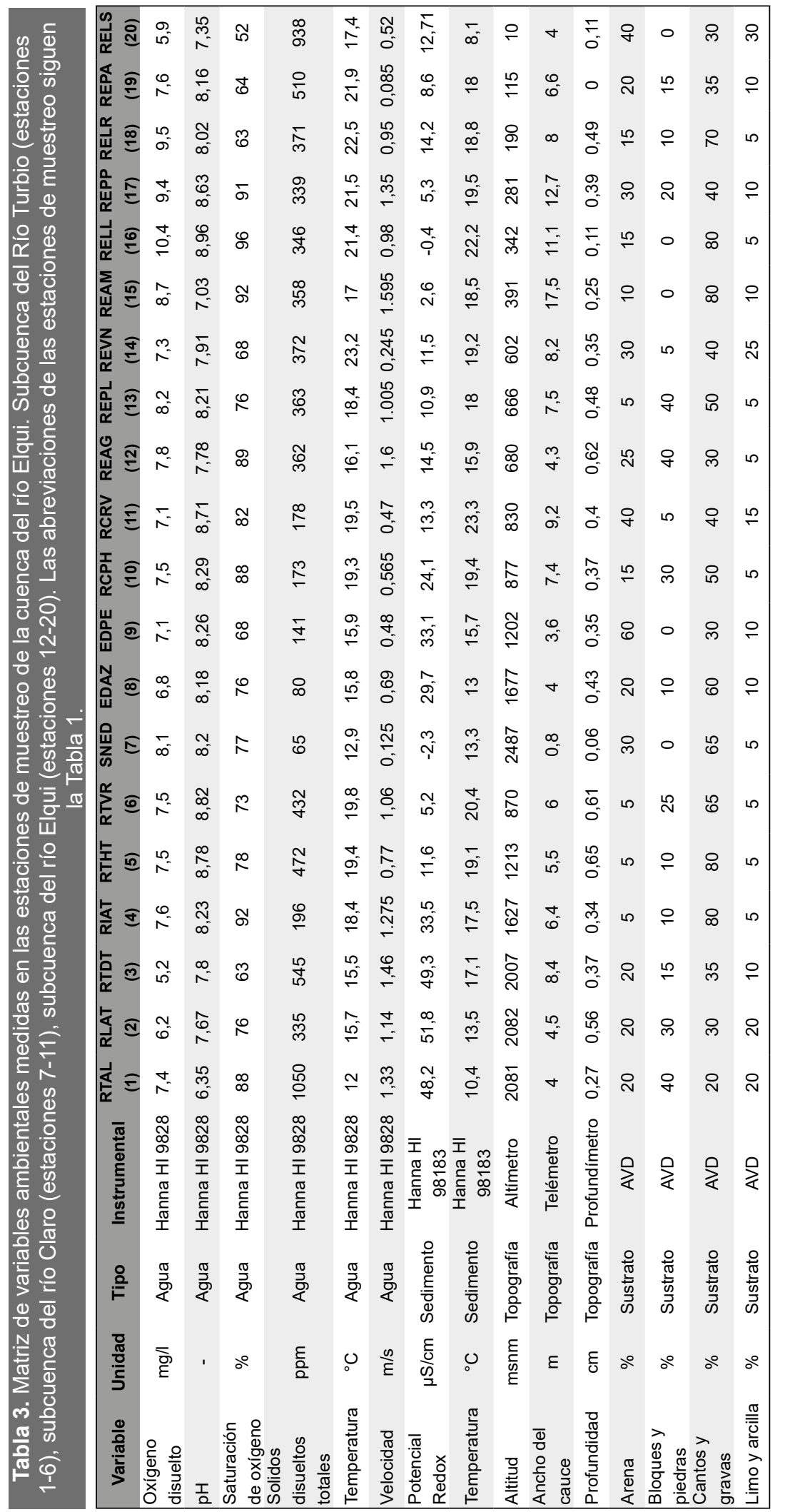




\section{Resultados}

Se registró una riqueza de 51 especies de plantas vasculares acuáticas y una carófita para la cuenca del río Elqui, de las cuales dos son endémicas del Cono Sur, 23 exóticas y 27 nativas (Tabla 2). En cuanto a los hábitos de crecimiento, aparecen dos flotantes libres, dos natantes, seis sumergidas y 42 emergentes. La familia mejor representada fue Cyperaceae con nueve especies, en tanto que a nivel de géneros destacan Hydrocotyle y Juncus, con tres especies cada uno. Las especies con mayor valor de importancia son Nasturtium officinale (Brassicaceae), Eleocharis pachycarpa (Cyperaceae) y Equisetum giganteum (Equisetaceae; Tabla 4). La mayor riqueza de especies se registró en las estaciones EDAZ (20 especies) y REPA (20 especies), en tanto que la menor se obtuvo en RTAL (3 especies; Fig. 2). La mayor cobertura del área independientemente de las especies ocurrió en la estación REPP (100\% de cobertura), en tanto que la menor fue registrada en RTAL (6,9 \%; Fig. 2). Los promedios de riqueza y abundancia para la cuenca del río Elqui fueron de 11,5 especies y $65,7 \%$ de cobertura, respectivamente.
A nivel de subcuencas, el río Turbio presenta una riqueza promedio de 4,8 especies y una cobertura promedio de $29,9 \%$. Cinco especies son exóticas y 10 nativas. Los hábitos de crecimiento se componen de dos plantas sumergidas y 13 emergentes. Se registró una especie que crece exclusivamente en la subcuenca de río Turbio (Juncus stipulatus; Tabla 4) y el mayor valor de importancia fue de Equisetum giganteum. La subcuenca del río Claro exhibe una riqueza promedio de 14,8 especies y una cobertura promedio de $80,8 \%$. Dos especies son endémicas del Cono Sur, 16 exóticas y 20 nativas. Los hábitos de crecimiento se conforman de dos especies natantes, dos sumergidas y 34 emergentes. Se registraron 11 especies que habitan exclusivamente en la subcuenca del río Claro, de las cuales siete sólo se encuentran en SNED (Tabla 4), el mayor valor de importancia fue de Apium nodiflorum. Finalmente, la subcuenca del río Elqui presenta una riqueza promedio de 14,2 especies y una abundancia promedio de $81,3 \%$ de cobertura. Una especie es endémica del Cono Sur, 17 son nativas y 19 exóticas. Los hábitos de crecimiento se componen de dos especies flotantes libres, dos natantes, seis sumergidas y 27 emergentes.

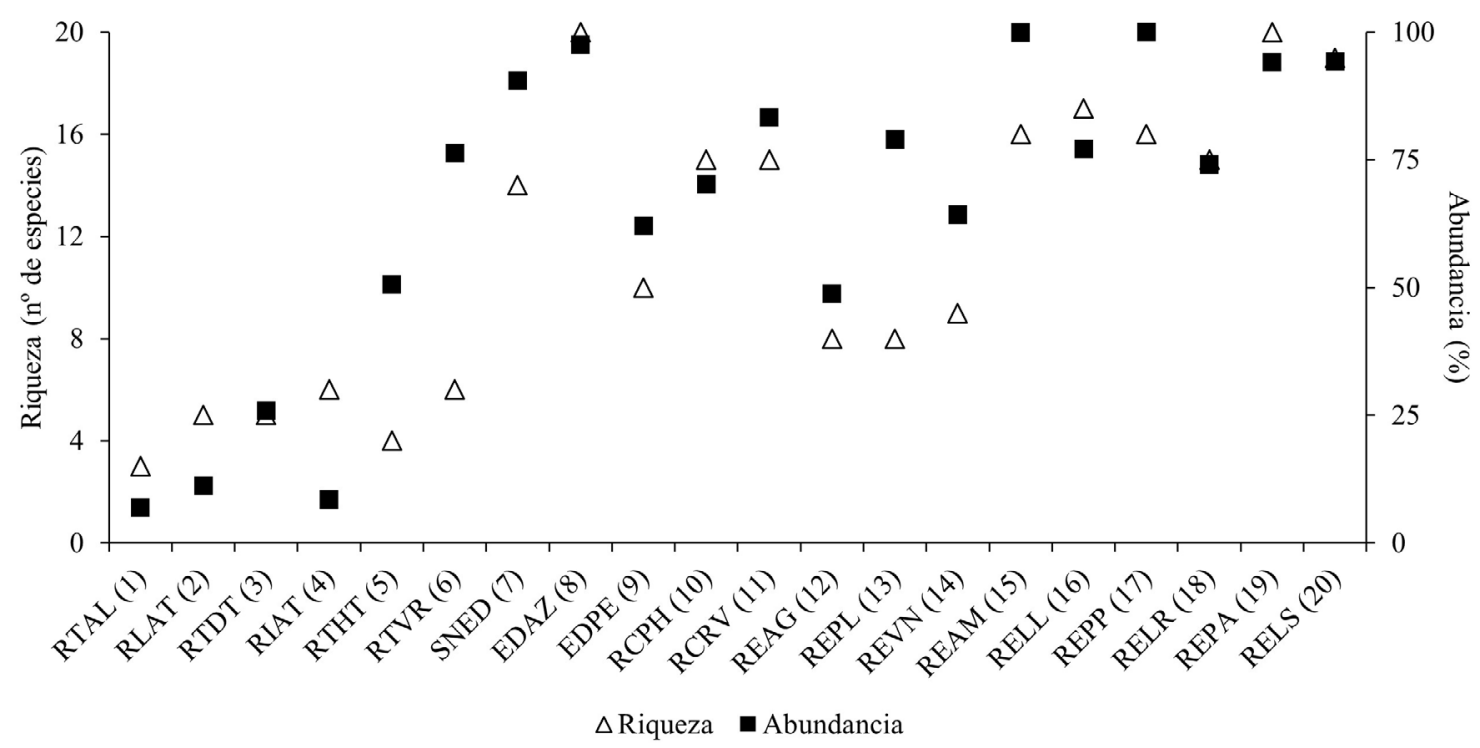

Fig. 2. Riqueza y abundancia de especies por estación de muestreo. Subcuenca del río Turbio (estaciones 1-6), subcuenca del río Claro (estaciones 7-11), subcuenca del río Elqui (estaciones 12-20). Las abreviaciones de las estaciones de muestreo siguen la Tabla 1. 


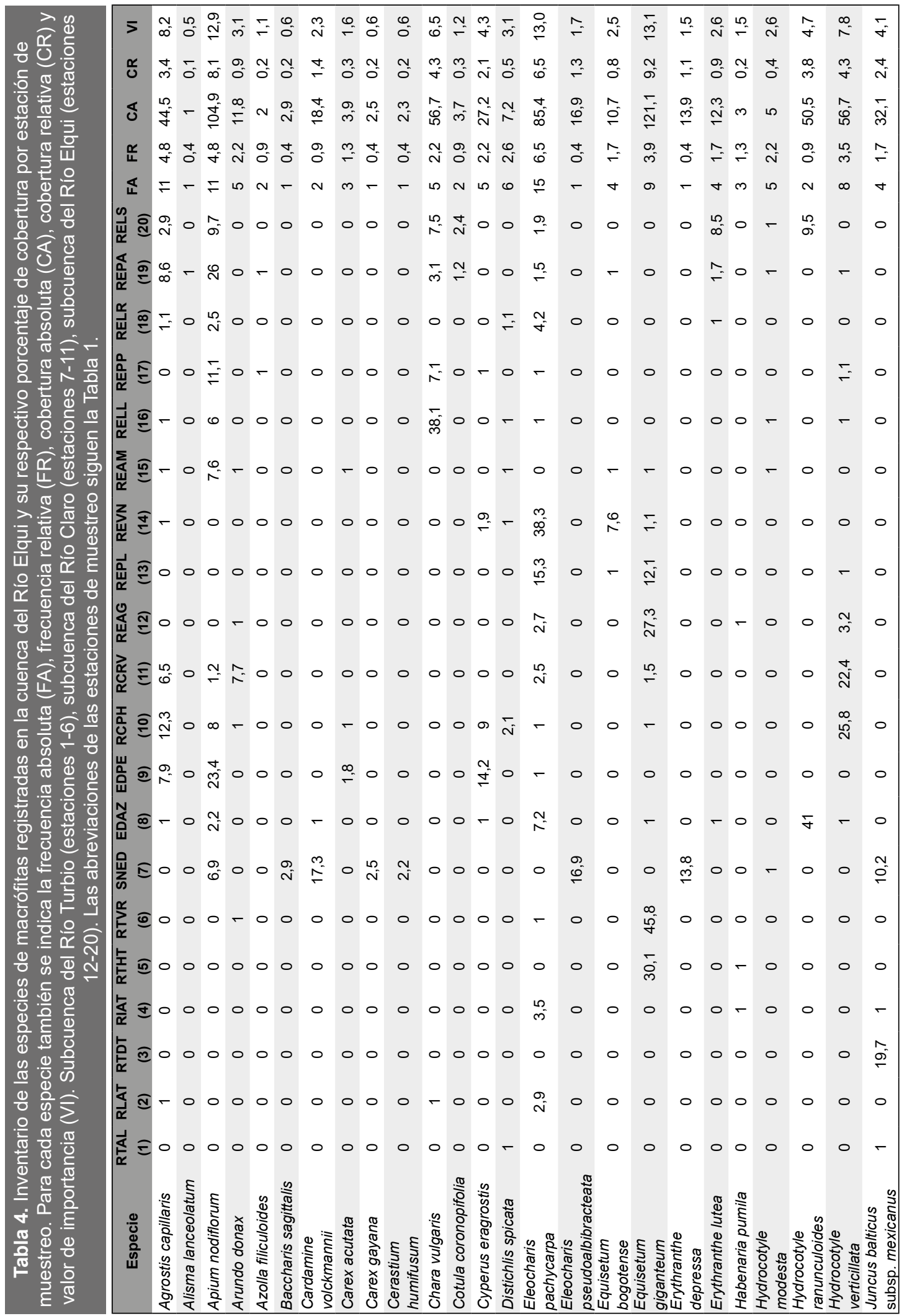




\begin{tabular}{|c|c|c|c|c|c|c|c|c|c|c|c|c|c|c|c|c|c|c|c|c|c|c|c|c|c|c|c|}
\hline 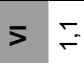 & $=\stackrel{L}{=} \stackrel{0}{=}$ & $\begin{array}{l}0 \\
0 \\
0\end{array}$ & $\begin{array}{l}\circ \\
\infty\end{array}$ & $\stackrel{\sim}{=}$ & $\begin{array}{l}10 \\
0 \\
0\end{array}$ & $\widehat{\infty}$ & $\begin{array}{l}10 \\
0 \\
0\end{array}$ & $\stackrel{\sigma}{m}$ & $\begin{array}{l}10 \\
0 \\
0\end{array}$ & 每 & $\bar{\sigma}$ & के & $\stackrel{\sim}{\sim}$ & $\stackrel{\circ}{\sim}$ & $\begin{array}{l}10 \\
0\end{array}$ & $\stackrel{+}{\circ}$ & $\begin{array}{l}10 \\
0 \\
0\end{array}$ & $\stackrel{10}{\sim}$ & $\stackrel{n}{\sim}$ & $\stackrel{n}{\sim}$ & $\begin{array}{c}\infty \\
\omega^{n}\end{array}$ & $\stackrel{\nabla}{\circ}$ & $m^{m}$ & $\stackrel{\cong}{=}$ & ㅇ. & $\stackrel{\Phi}{m}$ & \\
\hline N & $\begin{array}{cc}N & 0 \\
0 & 0\end{array}$ & $\overline{0}$ & $\stackrel{\infty}{\infty}$ & ப் & $\overline{0}$ & $\underset{\omega}{0}$ & $\bar{\circ}$ & $\ddot{\circ}$ & $\bar{\circ}$ & $\overline{0}$ & $\stackrel{0}{0}$ & $\stackrel{0}{N}$ & $\stackrel{m}{r}$ & $\ddot{0}$ & $\overline{0}$ & $\stackrel{\infty}{\forall}$ & $\bar{\circ}$ & $\tilde{O}$ & $\dddot{N}$ & $\tilde{O}$ & $\stackrel{g}{-}$ & $\bar{f}$ & $\bar{m}$ & $\stackrel{\infty}{\circ}$ & $\hat{\sigma}$ & 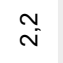 & \\
\hline$N$ & $N \stackrel{\Omega}{N}$ & $r$ & $\begin{array}{c}m \\
0 \\
0\end{array}$ & L & $\tau$ & 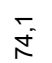 & 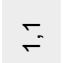 & $\begin{array}{l}\text { M } \\
\stackrel{0}{N}\end{array}$ & $\stackrel{\infty}{\leftarrow}$ & \ulcorner & $\stackrel{+}{\infty}$ & స̃ & $\begin{array}{l}0 \\
0 \\
0\end{array}$ & $\stackrel{t}{N}$ & $\stackrel{\infty}{\sim}$ & ๙ి & $r$ & $m$ & $m$ & $m$ & ָे & $\begin{array}{l}n \\
\tilde{N}^{0}\end{array}$ & $\frac{\pi}{\dot{g}}$ & $\stackrel{m}{\stackrel{0}{\circ}}$ & $\stackrel{\infty}{\underset{N}{N}}$ & $\stackrel{+}{\sim}$ & $\frac{10}{m}$ \\
\hline ه্ & ס & $\stackrel{\Delta}{\circ}$ & $\stackrel{\sim}{\sim}$ & $\stackrel{m}{\sim}$ & $\stackrel{\forall}{\Delta}$ & $\bar{m}$ & $\stackrel{+}{0}$ & $\stackrel{m}{\forall}$ & $\stackrel{\Delta}{\Delta}$ & $\stackrel{\Delta}{\circ}$ & $\begin{array}{l}n \\
\tilde{m}\end{array}$ & $\stackrel{\Delta}{N}$ & O) & $\stackrel{\Delta}{0}$ & $\stackrel{+}{\circ}$ & $\begin{array}{l}0 \\
60\end{array}$ & $\stackrel{\Delta}{\Delta}$ & $\stackrel{m}{\sim}$ & $\stackrel{m}{\sim}$ & $\stackrel{m}{\sim}$ & ભ̆ & $\stackrel{m}{\sim}$ & $\stackrel{N}{N}$ & $\stackrel{\circ}{0}$ & $\stackrel{m}{\sigma}$ & $\hat{\sigma}$ & \\
\hline$N$ & $N \sim$ & - & 10 & $m$ & - & $\wedge$ & - & 우 & - & - & $\infty$ & 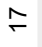 & N & - & - & $\stackrel{m}{\longrightarrow}$ & - & $m$ & $m$ & $m$ & $\sigma$ & $m$ & 10 & $\sim$ & 으 & $\nabla$ & $\bar{N}$ \\
\hline ฮ્సે & 0 & $r$ & $\stackrel{\hat{N}}{\sim}$ & 0 & 0 & 0 & 0 & $\hat{\sigma}$ & $\stackrel{\infty}{=}$ & 0 & 0 & - & $\bar{\sigma}$ & 0 & 0 & 0 & 0 & 0 & 0 & - & m & 0 & $\stackrel{\circ}{\circ}$ & - & - & 0 & \\
\hline 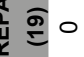 & 0 & 0 & $\overline{\tilde{N}}$ & - & 0 & 0 & 0 & $\stackrel{m}{\stackrel{9}{6}}$ & 0 & 0 & - & - & 0 & 0 & 0 & $\stackrel{m}{\sim}$ & 0 & 0 & 0 & - & $\stackrel{N}{=}$ & 0 & 0 & 0 & - & 0 & \\
\hline$\stackrel{\infty}{=} 0$ & 0 & 0 & $\overline{\dot{v}}$ & 0 & 0 & $\tau$ & 0 & $\stackrel{\infty}{F}$ & 0 & 0 & $\stackrel{\nabla}{\sim}$ & - & 0 & 0 & 0 & 0 & 0 & $r$ & 0 & 0 & $\check{\sigma}$ & 0 & $\stackrel{\check{I}}{\leftarrow}$ & 0 & $\stackrel{\nabla}{\sim}$ & $\stackrel{\infty}{-}$ & \\
\hline & 0 & 0 & $\begin{array}{l}m \\
\infty\end{array}$ & 0 & 0 & $\sigma$ & 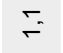 & $\overline{\dddot{g}}$ & 0 & 0 & - & $\stackrel{m}{=}$ & 0 & 0 & 0 & 0 & 0 & 0 & 0 & 0 & - & 0 & $\dot{m}$ & 0 & $\stackrel{\stackrel{n}{m}}{m}$ & क) & \\
\hline$\stackrel{0}{0}$ & 0 & 0 & 0 & 0 & 0 & $\stackrel{N}{O}$ & 0 & $r$ & 0 & 0 & - & - & 0 & 0 & 0 & $r$ & 0 & 0 & 0 & - & $r$ & 0 & $\hat{\sigma}$ & 0 & - & 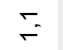 & \\
\hline & 0 & 0 & 0 & $\stackrel{\cong}{=}$ & 0 & $\stackrel{+}{\stackrel{N}{\leftarrow}}$ & 0 & స్ల & 0 & 0 & 0 & $r$ & 0 & 0 & 0 & $\stackrel{\Delta}{\sim}$ & 0 & 0 & 0 & 0 & 0 & 0 & - & 0 & $\stackrel{m}{\sigma}$ & $\begin{array}{l}0 \\
\stackrel{0}{\circ}\end{array}$ & \\
\hline & 0 & 0 & 0 & 0 & 0 & 0 & 0 & 0 & 0 & 0 & 0 & - & 0 & 0 & 0 & $\begin{array}{l}0 \\
0 \\
0\end{array}$ & 0 & 0 & 0 & 0 & 0 & $\hat{6}$ & 0 & 0 & 0 & 0 & \\
\hline 20 & 0 & 0 & 0 & 0 & 0 & 0 & 0 & 0 & 0 & 0 & 0 & O̊ & 0 & 0 & 0 & - & 0 & 0 & 0 & 0 & 0 & $\begin{array}{l}m \\
\infty^{\circ}\end{array}$ & 0 & ๙̃ & 0 & 0 & \\
\hline$\underline{y} 0$ & 0 & 0 & 0 & 0 & 0 & 0 & 0 & 0 & 0 & 0 & 0 & - & 0 & 0 & 0 & $\underset{\infty}{N}$ & 0 & 0 & 0 & 0 & $\bar{f}$ & 0 & 0 & 0 & 0 & 0 & \\
\hline$E-$ & -0 & 0 & 0 & 0 & 0 & $\stackrel{\sigma}{\tilde{N}}$ & 0 & - & 0 & 0 & - & - & 0 & 0 & 0 & $N$ & 0 & 0 & - & 0 & 0 & $\stackrel{\dot{\sigma}}{\circ}$ & 0 & 0 & - & 0 & \\
\hline 0 & 0 & 0 & - & 0 & 0 & 0 & 0 & $\stackrel{\infty}{N}$ & 0 & 0 & - & - & 0 & 0 & 0 & in & 0 & 0 & 0 & 0 & 0 & 0 & 0 & 0 & - & 0 & \\
\hline 0 & 0 & 0 & 0 & 0 & 0 & 0 & 0 & $\hat{\sigma}$ & 0 & 0 & - & - & 0 & 0 & 0 & - & 0 & $r$ & 0 & 0 & 0 & 0 & 0 & 0 & 0 & 0 & \\
\hline 0 & 0 & 0 & 0 & $\stackrel{\infty}{\sim}$ & - & $\stackrel{\varphi}{\circ}$ & 0 & $\stackrel{\nabla}{r}$ & 0 & 0 & - & 0 & 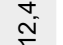 & 0 & $\stackrel{\infty}{\sim}$ & 0 & - & $r$ & - & 0 & 0 & 0 & 0 & 0 & 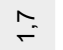 & 0 & \\
\hline 0 & 0 & 0 & 0 & 0 & 0 & 0 & 0 & 0 & 0 & - & 0 & $r$ & 0 & $\stackrel{m}{N}$ & 0 & 0 & 0 & 0 & 0 & 0 & ก̃ & 0 & 0 & 0 & 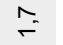 & 0 & \\
\hline & 0 & 0 & 0 & 0 & 0 & 0 & 0 & 0 & 0 & 0 & 0 & $\hat{s}$ & 0 & 0 & 0 & $\frac{\infty}{F}$ & 0 & 0 & - & 0 & 0 & 0 & 0 & 0 & 0 & 0 & \\
\hline 0 & 0 & 0 & 0 & 0 & 0 & 0 & 0 & 0 & 0 & 0 & 0 & $\stackrel{L}{=}$ & 0 & 0 & 0 & $\stackrel{-}{\leftarrow}$ & 0 & 0 & 0 & 0 & 0 & 0 & 0 & 0 & 0 & 0 & \\
\hline- & -0 & 0 & 0 & 0 & 0 & 0 & 0 & 0 & 0 & 0 & 0 & - & 0 & 0 & 0 & $r$ & 0 & 0 & 0 & 0 & 0 & 0 & 0 & 0 & 0 & 0 & \\
\hline 20 & 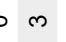 & 0 & 0 & 0 & 0 & 0 & 0 & 0 & 0 & 0 & 0 & $\stackrel{\sim}{\sim}$ & 0 & 0 & 0 & - & 0 & 0 & 0 & 0 & - & 0 & 0 & 0 & 0 & 0 & \\
\hline 0 & 0 & 0 & 0 & 0 & 0 & $\stackrel{m}{\sim}$ & 0 & 0 & 0 & 0 & 0 & 0 & 0 & 0 & 0 & 0 & 0 & 0 & 0 & 0 & 10 & 0 & 0 & 0 & 0 & 0 & \\
\hline 0 & 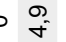 & 0 & 0 & 0 & 0 & 0 & 0 & 0 & 0 & 0 & 0 & 0 & 0 & 0 & 0 & 0 & 0 & 0 & 0 & 0 & 0 & 0 & 0 & 0 & 0 & 0 & \\
\hline 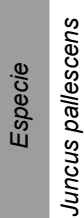 & 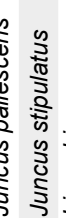 & 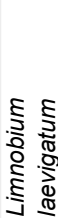 & 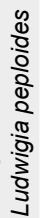 & 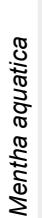 & 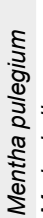 & 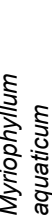 & 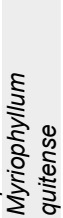 & 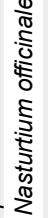 & 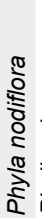 & 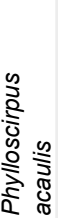 & 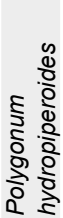 & 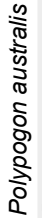 & 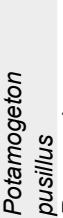 & 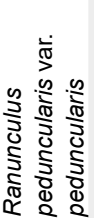 & 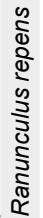 & 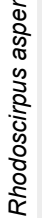 & 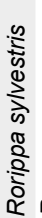 & 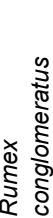 & 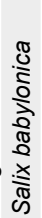 & 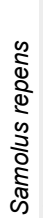 & 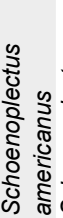 & 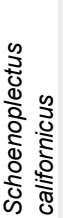 & 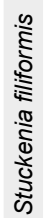 & 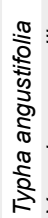 & 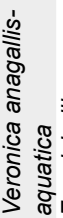 & 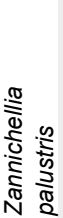 & \\
\hline
\end{tabular}




\section{J. Urrutia-Estrada y M. Romero-Mieres - Macrófitas de la cuenca del río Elqui}

Se registraron 11 especies que crecen sólo en la subcuenca del río Elqui y el mayor valor de importancia fue de Nasturtium officinale.

El nMDS muestra la ordenación espacial de cada una de las estaciones de muestreo con base en su composición florística (Fig. 3). Por su parte, el ANOSIM revela la existencia de sutiles pero significativas diferencias en la composición florística que presentan las subcuencas ( $\mathrm{R}$ global: 0,300; p: 0,008). En tanto que las comparaciones pareadas indican que dichas diferencias ocurren en las confrontaciones río Turbio - río Elqui (R: 0,428; p: 0,004) y río Turbio - río Claro (R: 0,363; p: 0,019).

La conjugación de la matriz ambiental (Tabla 3) y la matriz biológica (Tabla 4) en el análisis BioEnv, muestra que el conjunto de variables ambientales que determina de mejor manera la composición florística de la cuenca del río Elqui, está conformado por: altitud, sólidos suspendidos totales, potencial redox y profundidad (R: 0,524 ; p: 0,01).

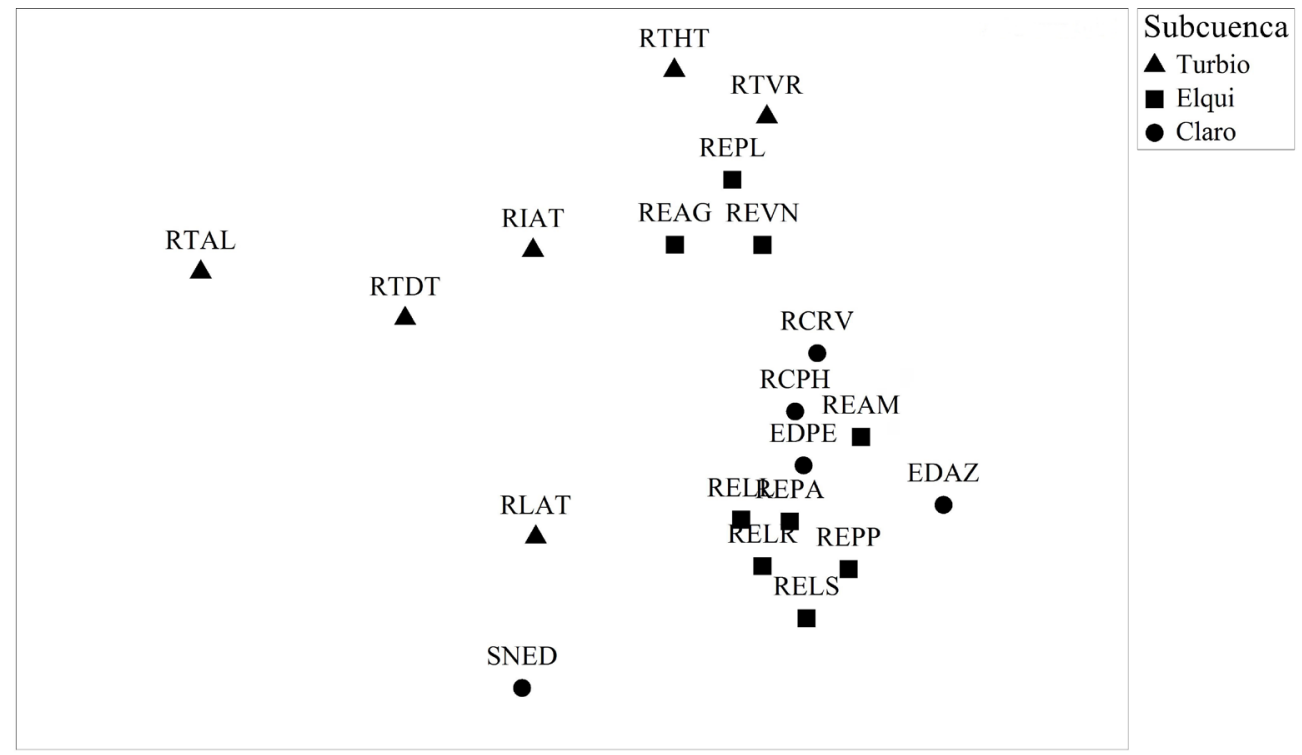

Fig. 3. Análisis de escalamiento multidimensional no-métrico (nMDS) para las estaciones de muestreo del área de estudio. Las abreviaciones de las estaciones de muestreo siguen la Tabla 1.

\section{Discusıón}

Si bien la subcuenca del río Claro presenta una riqueza de especies levemente mayor que la subcuenca del río Elqui, es en este último tramo donde se exhibe una mayor abundancia de especies en términos de cobertura y una expresión completa del espectro biológico en cuanto a los hábitos de crecimiento de las macrófitas.

Los estudios relativos a macrófitas a nivel de cuenca hidrográfica son prácticamente nulos en Chile, e inexistentes en lo referido a la zona semiárida del país. Un intento por llenar este vacío de información corresponde al trabajo de Palma et al. (2013), quienes construyeron un listado de especies para cinco cuencas hidrográficas ubicadas en un gradiente árido-mediterráneo del país. Los datos recabados tuvieron como base principalmente informes técnicos devenidos de consultorías ambientales. En este sentido se informan cuatro especies de macrófitas para el río Loa, 20 para el río Limarí, 31 para el río Huasco, 36 para el río Cachapoal y 58 para el río Mataquito. Lo más cercano en el contexto nacional, en cuanto a distancia geográfica y tipo de información florística levantada, corresponden a trabajos desarrollados en cuerpos de agua lóticos menores en la Región de Valparaíso. Más específicamente en los esteros 
Limache, Marga-Marga, Reñaca y Viña del Mar, en donde se registraron 13, 41, 73 y 80 especies de macrófitas respectivamente (Palma et al., 1978; Palma et al., 1987; San Martín et al., 2001; Arancibia \& Araya, 2014). Cabe también mencionar un estudio llevado a cabo en el río Aconcagua (Región de Valparaíso), donde se determinó una riqueza de 20 especies de macrófitas (Arancibia, 2006). Esta diferencia en comparación con nuestros resultados viene dada principalmente por la menor superficie estudiada, ya que sólo se prospectó el área de la desembocadura del río Aconcagua. En el escenario sudamericano destacan los trabajos realizados en Brasil (França et al., 2003; Henry-Silva et al., 2010) y Argentina (Jocou \& Gandullo 2020). En dos de los tres estudios mencionados anteriormente se registró un gran número de especies, 121 en el estado de Bahía, Brasil (França et al., 2003) y 137 en los humedales del norte de la Patagonia, Argentina (Jocou \& Gandullo 2020). Las notorias diferencias de riqueza respecto del río Elqui probablemente se pueden atribuir a factores climáticos (Schlesinger \& Bernhardt 2013) o condiciones locales que afectan directamente a la presencia de macrófitas (Ramírez \& San Martín 2006). Además, se debe tener en cuenta la heterogeneidad ambiental y el mayor número de cursos de agua muestreados en ambos casos. Para el río Apodi-Mossoro (Brasil), HenrySilva et al. (2010) registraron una riqueza de 40 especies de macrófitas, siendo Cyperaceae una de las familias mejor representadas del estudio.

La diversidad florística encontrada en la cuenca del río Elqui es consecuencia de las importantes variaciones en las condiciones de hábitats, determinadas de manera conjunta por la variabilidad del relieve, la diferencia de altitud, la influencia de la salinidad marina (en las estaciones más bajas) y la heterogeneidad del sustrato alóctono acumulado por la actividad antrópica. La riqueza de macrófitas reportada en el presente estudio, representa alrededor del $10 \%$ de la flora acuática y palustre documentada para el país (Hauenstein, 2006; Ramírez \& San Martín, 2006). Tal porcentaje es muy importante, si se considera que el río Elqui presenta un flujo de agua no siempre constante, convirtiéndolo en un río de alta vulnerabilidad (Cepeda et al., 2008). A nivel de subcuencas, la mayor diferencia en cuanto a riqueza se presentó en el río Turbio, donde destaca el bajo número de especies en comparación al río Claro y al río Elqui. Esto podría tener su explicación en el desarrollo de actividades mineras que tuvieron lugar en las zonas más cercanas a la cabecera del río Turbio, lo que generó residuos que eventualmente pudieron afectar la calidad de vida de las plantas. Además, podrían estar influyendo otros factores medidos en el campo, tales como el mayor porcentaje de piedras en el sustrato, lo que dificulta el enraizamiento de las plantas (Ramírez \& San Martín, 2006) y los valores de sólidos disueltos totales, que de manera indirecta podrían estar indicando una menor calidad de agua en comparación a las otras dos subcuencas.

A pesar de existir un dominio considerable de las especies de hábito emergente, es importante señalar que el espectro biológico se encuentra completo, exhibiendo representantes de todas las formas de crecimiento propias de las macrófitas. En este sentido, cabe destacar la presencia de seis especies sumergidas y dos flotantes libres, lo cual es relevante tratándose de un sistema fluvial, ya que estas formas de crecimiento se desarrollan de mejor manera en sistemas lénticos (San Martín et al., 2001; Urrutia et al., 2017a). Con relación a esto, la velocidad del flujo de agua es un parámetro que incide de manera importante en el crecimiento y la distribución de las especies acuáticas (Janauer et al., 2010). Además, determina la permanencia de una planta, el éxito de colonización en ciertos hábitats (Riis et al., 2008) e influye en la composición de especies, biomasa, morfología y metabolismo de las plantas (Chambers et al., 1991).

A nivel de familia, los resultados evidencian que Cyperaceae es la más importante de acuerdo con el número de especies, una situación esperable, ya que sus representantes se encuentran generalmente restringidos a ambientes húmedos, siendo común encontrarlos a orillas de cuerpos de agua lacustres, fluviales y como importantes componentes de la flora de humedales (Smith et al., 2004). La destacada presencia de esta familia en los ambientes acuáticos se debe al desarrollo de rizomas y estolones que facilitan su propagación vegetativa (França et al., 2003; Matias et al., 2003).

Mención especial merecen las especies exóticas, cuyo aporte en el presente estudio es relevante, en circunstancias que configuran más del $40 \%$ del espectro florístico. En este sentido, son las especies de hábito acuático las que presentan mayor potencial de convertirse en invasoras (Urrutia et al., 2017b; Urrutia-Estrada \& Hauenstein, 2021), en muchos casos incluso más que sus contrapartes terrestres 


\section{J. Urrutia-Estrada y M. Romero-Mieres - Macrófitas de la cuenca del río Elqui}

(Gordon et al., 2012). Los sistemas de aguas corrientes (fluviales), están entre los ambientes más invadidos a escala global (Hejda et al., 2015), probablemente debido a que el agua actúa como un buen agente dispersor de propágulos (Johansson \& Nilson, 1993). Lo anterior obedece a las múltiples estructuras heterogéneas que presentan los sistemas fluviales, caracterizados por altas tasas de conectividad, simplicidad en el transporte de propágulos y variedad de microhábitats (Gurnell et al., 2008).

La aparición de especies como Cotula coronopifolia y Phyla nodiflora en la estación más cercana al mar (RELS), da cuenta de la influencia de la salinidad en la zona de la desembocadura del río Elqui. En este sentido, los ambientes tipo estuarios generan una heterogeneidad en los propiedades físico-químicas del agua, lo que da como resultado la conformación de hábitats únicos e importantes para algunas especies de plantas (Mucina et al., 2006).

Si bien la latitud actúa como uno de los factores más importantes en determinar la riqueza de una comunidad (Stevens, 1989), la altitud también juega un rol importante (Lacoul \& Freedman, 2006; Murphy et al., 2019). Así lo refrenda el resultado del análisis Bio-Env, en donde esta última variable asoma como la más importante en configurar la comunidad de macrófitas de la cuenca del río Elqui. En este contexto, cabe señalar que la estación de muestreo con mayor elevación (SNED, $2487 \mathrm{msnm}$ ), muestra una configuración de especies algo distinta, lo que provoca un aislamiento florístico del resto del área de estudio (Fig. 3). Estos resultados se condicen de buena manera con antecedentes recientes, los cuales demuestran que si bien la altitud no es un buen predictor de la riqueza de especies en cuerpos de agua altoandinos (Fernández-Aláez et al., 2018), puede influir de manera importante en exhibir un ensamble de especies distinto al de las otras zonas de menor altitud. Esto último lo refuerza el registro exclusivo de Erythranthe depressa en SNED, una de las dos especies endémicas registradas en el presente estudio.

Las prospecciones florísticas por básicas que parezcan son de gran utilidad, ya que constituyen la base del conocimiento relativo a la biodiversidad, lo que posteriormente permite desarrollar estrategias de conservación y determinar las acciones a seguir en la gestión de los recursos naturales. La generación de este tipo de información es un aporte valioso en lo que concierne a la estructura y al funcionamiento de los ambientes fluviales, lo que repercutirá en un manejo más eficiente de los sistemas acuáticos continentales.

\section{Contribución DE LOS AUTORES}

JUE llevó a cabo el diseño muestral y realizó la colecta de datos en terreno. Ambos autores participaron en el análisis de datos y redacción del manuscrito.

\section{Agradecimientos}

Al proyecto ANID FB210006 y a la licitación pública 612228-9-LE19, de la Seremi del Medio Ambiente de la Región de Coquimbo, Chile.

\section{Bibliografía}

ARANCIBIA, J. 2006. Flora vascular en la desembocadura del Río Aconcagua, V Región Chile. An. Mus. Hist. Nat. Valpso. 25: 34-46.

ARANCIBIA, J. \& M. ARAYA. 2014. Diversidad, abundancia y distribución de la flora vascular del estero de Viña del Mar, región de Valparaiso, Chile. An. Mus. Hist. Nat. Valpso. 27: 15-27.

BAEZA, M., R. RODRÍGUEZ \& O. TORO-NÚÑEZ. 2019. Flora vascular de la Laguna Avendaño, Provincia de Diguillín, Chile. Gayana Bot. 76: 74-83. http://dx.doi.org/10.4067/S0717-66432019000100074

CEPEDA, J., C. ZULETA \& F. LÓPEZ. 2008. Síntesis: los sistemas naturales de la cuenca del Río Elqui en el contexto del cambio climático. En CEPEDA, P. (ed.). Los sistemas naturales de la cuenca del Río Elqui (Región de Coquimbo, Chile). Vulnerabilidad y cambio del clima, pp. 337-379. Ediciones Universidad de La Serena, La Serena.

CHAMBERS, P., E. PREPAS, H. HAMILTON \& M. BOTHWELL. 1991. Current velocity and its effect on aquatic macrophytes in flowing waters. Ecol. Appl. 1: 249-257. https://doi.org/10.2307/1941754

CLARKE, K. \& R. GORLEY. 2006. Plymouth routines in multivariate ecological research. Primer-E. Plymouth Marine Laboratory, London. 
CONTE, A. 1986. Vulnerabilidad a los eventos naturales catastróficos de los valles Elqui, Limari y Choapa, IV region. Revista Geográfica de Chile Terra Australis. 29: 103-130.

DAVIES, B., M. THOMS, K. WALKER, J. O'KEEFFE \& J. GORE. 1994. Drylands rivers: Their ecology, conservation and management. In CALLOW, P. \& G. PETTS (eds.), The river handbook, pp. 484-511. Blackwell, Oxford. https://doi.org/10.1002/9781444313871.ch25

DESMET, N., S. VAN BELLEGHEM, P. SEUNTJENS, T. BOUMA, K. BUIS \& P. MEIRE. 2011. Quantification of the impacts of macrophytes on oxygen dynamics and nitrogen retention in a vegetated lowland river. Phys. Chem. Earth. 36: 479-489. https://doi.org/10.1016/j.pce.2008.06.002

DITOMASO, J. \& E. HEALY. 2003. Aquatic and riparian weeds of the west. University of California, Oakland.

FERNÁNDEZ-ALÁEZ, C., M. FERNÁNDEZ-ALÁEZ, F. GARCÍA-CRIADO \& J. GARCÍA-GIRÓN. 2018. Environmental drivers of aquatic macrophyte assemblages in ponds along an altitudinal gradient. Hydrobiologia 812: 79-98. https://doi.org/10.1007/s10750-016-2832-5

FRANÇA, F., E. MELO, A. GÓES, D. ARAÚJO, M. BEZERRA, H. RAMOS, I. CASTRO \& D. GOMES. 2003. Flora vascular de açudes de uma região do semi-árido da Bahia, Brasil. Acta Bot. Bras. 17: 549-559.

https://doi.org/10.1590/S0102-33062003000400008

FUENTES, N., P. SÁNCHEZ, A. PAUCHARD, J. URRUTIA, L. CAVIERES \& A. MARTICORENA. 2014. Plantas invasoras del centro-sur de Chile: una guía de campo. Laboratorio de Invasiones Biológicas, Universidad de Concepción, Concepción.

GÓMEZ, R., M. VIDAL-ABARCA \& M. SUÁREZ. 2001. Importance of the subsurface-surface water interaction in the wetland structure and dynamic in arid and semiarid areas. In: GRIEBLER, C., D. DANIELOPOL, J. GIBERT, H. NACHTNEBEL \& J. NOTENBOOM (eds.), Groundwater ecology. A tool for management of water resources, pp. 317-322. European Commission-Environment and Climate Programe, Belgium.

GORDON, D., C. GANTZ, C. JERDE, W. CHADDERTON, R. KELLER \& P. CHAMPION. 2012. Weed risk assessment for aquatic plants: Modification of a New Zealand system for the United States. PLoS ONE 7: e40031. https://doi.org/10.1371/journal.pone.0040031
GUEVARA, S., J. OYARZÚN \& H. MATURANA. 2006. Geoquímica de las aguas del Río Elqui y de sus tributarios en el período 1975-1995: factores naturales y efecto de las explotaciones mineras en sus contenidos de Fe, Cu y As. Agric. Téc. 66: 57-69. http://dx.doi.org/10.4067/S0365-28072006000100007

GURNELL, A., K. THOMPSON, J. GOODSON \& H. MOGGRIDGE. 2008. Propagule deposition along river margins: linking hydrology and ecology. $J$. Ecol. 96: 553-565.

https://doi.org/10.1111/j.1365-2745.2008.01358.x

HAUENSTEIN, E., F. BARRIGA \& P. DE LOS RÍOS. 2011. Macrophytes assemblages in mountain lakes of Huerquehue National Park $\left(39^{\circ} \mathrm{S}\right.$, Araucanía Region, Chile). Lat. Am. J. Aquat. Res. 39: 593-599. https://doi.org/10.3856/vol39-issue3-fulltext-19

HAUENSTEIN, E. \& J. URRUTIA-ESTRADA. 2018. Flora hidrófila de La Araucanía. En HERNÁNDEZ, M., A. BEJCEK \& A. MUÑOZ-PEDREROS (eds.), Humedales. La importancia de su valorización y conservación en la Región de La Araucanía, pp. 1526. CEA Ediciones. Ministerio del Medio Ambiente, Temuco.

HEJDA, M., M. CHYTRÝ, J. PERGL \& P. PYŠEK, 2015. Native-range habitats of invasive plants: are they similar to invaded range habitats and do they differ according to the geographical direction of invasion? Divers. Distrib. 21: 312-321. https://doi.org/10.1111/ddi.12269

HENRY-SILVA, G., R. MOURA \& L. DANTAS. 2010. Richness and distribution of aquatic macrophytes in Brazilian semi-arid aquatic ecosystems. Acta Limnol. Bras. 22: 147-156. https://doi.org/10.4322/actalb.02202004

JANAUER, G., U. SCHMIDT-MUMM \& B. SCHMIDT. 2010. Aquatic macrophytes and water current velocity in the Danube River. Ecol. Eng. 36: 11381145. https://doi.org/10.1016/j.ecoleng.2010.05.002

JOCOU, A. \& R. GANDULLO. 2020. Diversidad de plantas vasculares de los humedales de la Norpatagonia (Argentina). Rev. Mus. Argentino Cienc. Nat. 22: 131-154.

JOHANSSON, M. \& C. NILSSON. 1993. Hydrochory, population dynamics and distribution of the clonal aquatic plant Ranunculus lingua. J. Ecol. 81: 81-91. https://doi.org/10.2307/2261226

LACOUL, P. \& B. FREEDMAN. 2006. Relationships between aquatic plants and environmental factors along a steep Himalayan altitudinal gradient. Aquat. Bot. 84: 3-16. https://doi.org/10.1016/j.aquabot.2005.06.011 


\section{J. Urrutia-Estrada y M. Romero-Mieres - Macrófitas de la cuenca del río Elqui}

MADSEN, J., P. CHAMBERS, W. JAMES, E. KOCH \& D. WESTLAKE. 2001. The interaction between water movement, sediment dynamics and submersed macrophytes. Hydrobiologia 444: 71-84.

https://doi.org/10.1023/A:1017520800568

MATIAS, L., E. AMADO \& E. NUNES. 2003. Macrófitas aquáticas da lagoa de Jijoca de Jericoacoara, Ceará, Brasil. Acta Bot. Bras. 17: 623-631. https://doi.org/10.1590/S0102-33062003000400015

MUCINA, L., J. ADAMS, I. KNEVEL, M. RUTHERFORD, M. POWRIE, J. BOLTON, H. VAN DER MERWE, J. ANDERSON, T. BORNMAN, A. LE ROUX \& J. JANSSEN. 2006. Coastal vegetation of South Africa. In: MUCINA, L. \& M. RUTHERFORD (eds.), The vegetation of South Africa, Lesotho and Swaziland, pp. 658-697. SANBI, Pretoria.

MUELLER-DOMBOIS, D. \& H. ELLENBERG. 1974. Aims and methods of vegetation ecology. John Wiley \& Sons, New York.

MURPHY, K., A. EFREMOV, T. DAVIDSON, E. MOLINA-NAVARRO, K. FIDANZA, T. CRIVELARI, P. CHAMBERS, J. TAPIA, S. VARANDAS, I. SPRINGUEL, M. KENNEDY, R. MORMUL, E. DIBBLE, D. HOFSTRA, B. LUKÁCS, D. GEBLER, L. BAASTRUP-SPOHR \& J. URRUTIA-ESTRADA. 2019. World distribution, diversity and endemism of aquatic macrophytes. Aquat. Bot. 158.

https://doi.org/10.1016/j.aquabot.2019.06.006

PALMA, A., J. GONZÁLEZ-BARRIENTOS, C. REYES

\& R. RAMOS-JILIBERTO. 2013. Biodiversidad y estructura comunitaria de ríos en las zonas árida, semiárida y mediterránea-norte de Chile. Rev. Chil. Hist. Nat. 86: 1-14. http://dx.doi.org/10.4067/S0716-078X2013000100001

PALMA, B., F. SAIZ \& C. PIZARRO. 1978. Variaciones de la taxocenosis de hidrófitas en el curso del estero Limache. An. Mus. Hist. Nat. Valpso. 11: 61-67.

PALMA, B., C. SAN MARTÍN, M. ROSALES, L. ZUÑIGA \& C. RAMIREZ. 1987. Distribución espacial de la flora y la vegetación acuática y palustre del estero Marga-Marga en Chile Central. Anales del Instituto de Ciencias del Mar y Limnología de la Universidad Nacional Autónoma de México 14: 125-132.

RAMÍREZ, C., R. GODOY, D. CONTRERAS \& E. STEGMAIER. 1982. Guía de plantas acuáticas $y$ palustres valdivianas. Facultad de Ciencias, Universidad Austral de Chile. Valdivia.
RAMÍREZ, C. \& J. SAN MARTÍN. 1984. Hydrophilous vegetation of a coastal lagoon in central Chile. $J$. Ecol. Environ. 10: 93-110.

RAMÍREZ, C. \& C. SAN MARTÍN. 2006. Diversidad de macrófitos chilenos. En VILA, I., A. VELOSO, R. SCHLATTER \& C. RAMÍREZ (eds.), Macrófitas $y$ vertebrados de los sistemas límnicos de Chile, pp. 21-61. Editorial Universitaria, Santiago.

RAMÍREZ, C. \& C. SAN MARTÍN. 2018. Flora acuática. En FIGUEROA, A., J. ROVIRA, S. FLORES, C. TALA, R. AVILÉS, J. ORELLANA, J. FERREYRA, P DÍAZ \& A. ARMENDARIZ (eds.), Biodiversidad de Chile. Patrimonio y desafios, pp. 219-227. Ministerio del Medio Ambiente, Santiago.

RAMÍREZ, C., J. SAN MARTÍN, C. SAN MARTÍN \& D. CONTRERAS. 1987. Estudio florístico y vegetacional de la Laguna El Peral, Quinta Región de Chile. Rev. Geogr. Valpso. 18: 105-120.

RIIS, T., A. SUREN, B. CLAUSEN \& K. SANDJENSEN. 2008. Vegetation and flow regime in lowland streams. Freshwater Biol. 58: 1531-1543. https://doi.org/10.1111/j.1365-2427.2008.01987.x

RODRÍGUEZ, R. \& V. DELLAROSSA. 1998. Plantas vasculares acuáticas en la Región del Biobio. Universidad de Concepción, Concepción.

RODRÍGUEZ, R., C. MARTICORENA, D. ALARCÓN, C. BAEZA, L. CAVIERES, V. FINOT, N. FUENTES, A. KIESSLING, M. MIHOC, A. PAUCHARD, E. RUÍZ, P. SÁNCHEZ \& A. MARTICORENA. 2018. Catálogo de las plantas vasculares de Chile. Gayana Bot. 75: 1-430.

http://dx.doi.org/10.4067/S0717-66432018000100001

SAN MARTÍN, C., Y. PÉREZ, D. MONTENEGRO \& M. ÁLVAREZ. 2011. Diversidad, hábito y hábitat de macrófitos acuáticos en la Patagonia occidental (Región de Aisén, Chile). An. Inst. Patagon. 39: 23-41. http://dx.doi.org/10.4067/S0718-686X2011000100002

SAN MARTÍN, C., C. RAMÍREZ, J. SAN MARTÍN \& R. VILLASEÑOR. 2001. Flora y vegetación del estero Reñaca (V Región, Chile). Gayana Bot. 58: 31-46. http://dx.doi.org/10.4067/S0717-66432001000100004

SANDOVAL, V., C. RAMÍREZ, C. SAN MARTÍN, O. VIDAL, M. ÁLVAREZ, A. MARTICORENA \& Y. PÉREZ. 2016. Diversidad vegetal en las cuencas de los ríos Baker y Pascua (Aisén, Patagonia Chilena). Bosque 37: 243-253.

http://dx.doi.org/10.4067/S0717-92002016000200003

SCULTHORPE, C. 1967. The biology of aquatic vascular plants. Edward Arnold, London. https://doi.org/10.1126/science.160.3824.179-a 
SMITH, N., S. MORI, A. HENDERSON, D. STEVENSON \& S. HEALD. 2004. Flowering plants of the Neotropics. Princeton University Press, New Jersey. https://doi.org/10.2307/25065340

STEUBING, L., R. GODOY \& M. ALBERDI. 2002. Métodos de ecología vegetal. Editorial Universitaria, Santiago.

STEVENS, G. 1989. The latitudinal gradient in geographical range: how so many species coexist in the tropics. Am. Nat. 133: 240-256. https://doi.org/10.1086/284913

URRUTIA-ESTRADA, J \& E. HAUENSTEIN. 2021. Plantas acuáticas invasoras presentes en el Cono-Sur de Sudamérica. Bioinvasiones 8: 19-26.

URRUTIA, J., R. RIVERA, E. HAUENSTEIN \& P. DE LOS RÍOS. 2012. Uso de modelos nulos para explicar asociaciones de macrófitas en ambientes lénticos de la región de La Araucanía, Chile. Phyton 81: 7-13.

URRUTIA, J., P. SÁNCHEZ, A. PAUCHARD \& E. HAUENSTEIN. 2017a. Flora palustre $y$ acuática introducida en Chile. Laboratorio de
Invasiones Biológicas, Universidad de Concepción, Concepción.

URRUTIA, J., P. SÁNCHEZ, A. PAUCHARD \& E. HAUENSTEIN. 2017b. Plantas acuáticas invasoras presentes en Chile: distribución, rasgos de vida y potencial invasor. Gayana Bot. 74: 147-157. http://dx.doi.org/10.4067/S0717-66432017005000324

VIDAL-ABARCA, M., R. GÓMEZ \& M. SUÁREZ. 2004. Los ríos de las regiones semiáridas. Ecosistemas. 13: 16-28.

WETZEL, R. 2001. Limnology. Lake and river ecosystems. Academic Press, San Diego. https://doi.org/10.1086/380040

WIKUM, D. \& G. SCHANHOLTZER. 1978. Application of the Braun-Blanquet cover-abundance scale for vegetation analysis in land development studies. Environ. Manage. 2: 323-329. https://doi.org/10.1007/BF01866672

ZULOAGA, F., M. BELGRANO \& C. ZANOTTI. 2019. Actualización del catálogo de las plantas vasculares del cono sur. Darwiniana 7: 208-278.

https://doi.org/10.14522/darwiniana.2019.72.861 